\title{
Breve historia del tratamiento de la litiasis vesical
}

\author{
C. Sevilla Cecilia, X. Pascual García, H. Villavicencio Mavrich \\ Servicio de Urología. Fundació Puigvert. Barcelona.
}

Actas Urol Esp 2005; 29 (10): 923-926

\section{RESUMEN}

BREVE HISTORIA DEL TRATAMIENTO DE LA LITIASIS VESICAL

La litiasis vesical es una de las patologías más antiguas que conocemos. La historia de su tratamiento ha sido motivo de controversia en diferentes momentos. Desde las primeras tallas vesicales de Egipto o la India, hasta la litotricia de nuestros días se han producido muchos tratamientos intermedios. El objetivo de este escrito es conocer de manera somera algunos de estos logros y sus autores así como el desarrollo de los materiales que han contribuido a conseguirlo.

Palabras clave: Historia. Litiasis vesical. Litotricia. Talla vesical.

\section{ABSTRACT}

BRIEF HISTORY OF VESICAL LITHIASIS MANAGEMENT

Stones of the bladder are one of the oldest illness that we know. History of its treatment has been cause of discussion in different moments. Since first treatments in Egypt or India, until our extracorporeal shock waves lithotritia (ESWL), there have been many intermediate treatments. The objective of this work is to know a little about some of these advances and their authors, as well as the instruments that have made it possible.

Keywords: History. Bladder stones. Lithotrities. Tall bladder.

$\mathrm{L}$ a litiasis vesical es una de las patologías más antiguas que conocemos. Su tratamiento ha sido objeto de múltiples discusiones a lo largo de la historia. La litotricia por ondas de choque ha sido uno de los grandes avances en el tratamiento de la litiasis vesical.

\section{OBJETIVOS}

Realizar un análisis de la bibliografía desde los primeros datos de la cirugia litiásica hasta nuestros tiempos.

\section{MATERIAL Y MÉTODOS}

A través de artículos y textos originales intentamos hacer un recorrido histórico de la evolución del tratamiento de la litiasis vesical. Desde el antiguo Egipto a la litotricia extracorpórea por ondas de choque muchas y variadas han sido las opciones terapéuticas.

\section{DISCUSIÓN}

La litiasis vesical es una patología conocida desde la antigüedad. De hecho se han encontrado momias egipcias con litiasis de oxalato cálcico y estrumita ${ }^{11}$.
Sin embargo las primeras descripciones escritas a cerca de su diagnóstico y tratamiento las encontramos en textos hindis ("Sus ruta").

$\mathrm{El}$ primer litotomiota conocido fue Amonios de Alejandría en el 247 a.C. ${ }^{11}$

Por todos es conocida la frase de Hipócrates "No realizarás la operación de la piedra y la dejarás en manos expertas" (Fig.1); en aquellos momentos los conocimientos anatómicos no eran muy exactos y la práctica de esta técnica no siempre era realizada por médicos ${ }^{11}$.

No es hasta el 50 d.C. cuando Celso en su obra "De Medicina" describe el mal de la piedra y la técnica de litotomía en jóvenes de 9-14 años (Libro VII de "De Medicina").

Con posterioridad, Galeno (Fig. 2), en el 129 d.C. perfecciona los conocimientos anatómicos y en base a las descripciones de Celso y Aristóteles describe su técnica de litotomía ${ }^{11}$.

En el siglo X un médico árabe llamado Avulcasis realizó la primera litotricia endoscópica "ciega" mediante la introducción en la vejiga de un aparato llamado "mashaba rebilia" que fragmentaba la litiasis en su interior. En sus textos encontramos las descripciones completas del ins- 


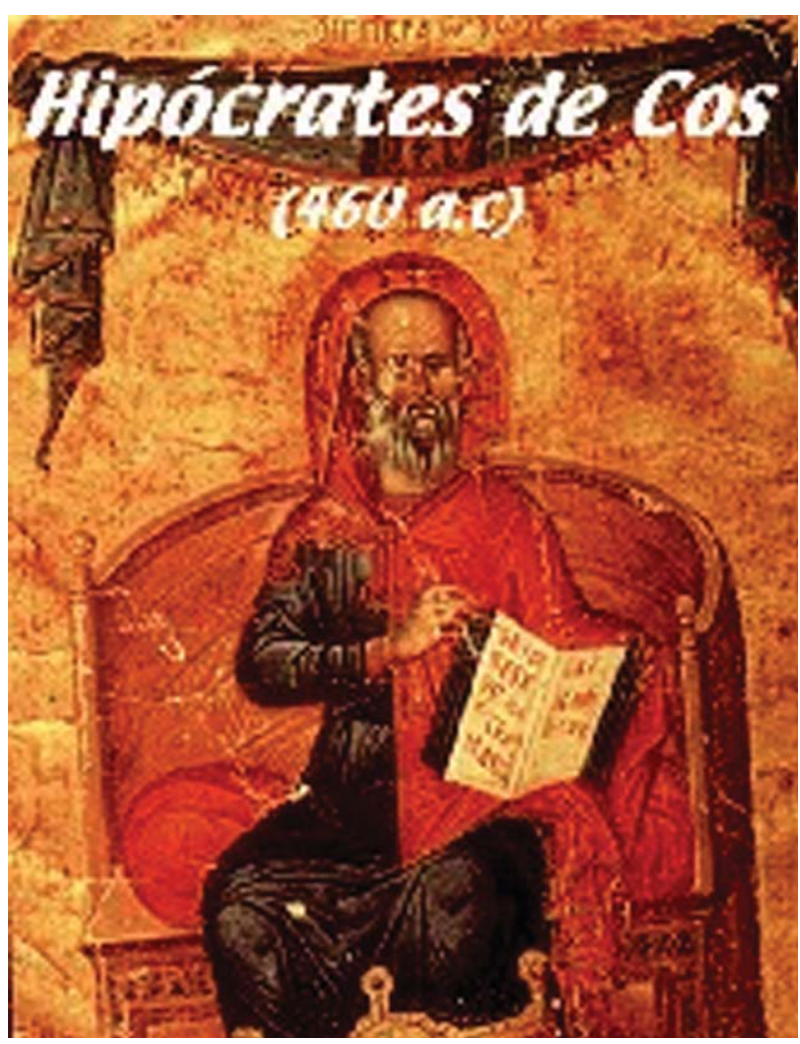

FIGURA 1. Hipócrates y su juramento aconsejaba no realizar la intervención de la piedra si no era realizada por expertos.

trumental necesario para realizar esta intervención así como la talla vesical. Este autor fue el primero en documentar la talla vesical en mujeres (cosa excepcional en el contexto de la sociedad árabe medieval) ${ }^{11}$.

Un español, Julián Gutiérrez, escribe en 1448 su obra "Cura de la piedra y dolor del yjada y/o cólico renal". Esta obra se divide en 5 tomos que tratan desde la prevención de las litiasis hasta el tratamiento de las mismas. Describe las causas de la litiasis en base a la teoría de los humores así como las trece causas de mal y veinticinco de buen pronóstico del mal de la piedra. En cuanto a la prevención, documentó 16 preceptos dietéticos básicos, así como evitar los accidentes del alma (furor, tristeza, exceso de trabajo, exceso de sexo, etc...) $)^{2,11}$ (Fig. 3).

También hace referencia al tratamiento del dolor de cólico utilizando hierbas como el opio o el beleño, o tratamientos para disolver las piedras (cenizas de escorpión o de liebre degollada, vísceras de cabrón picado etc..).

Años más tarde en 1521 Pierre Franco se hace un nombre como litotomista y describe por primera vez la técnica de litotomía suprapúbica, realizada a un joven al que no podía extraer la litiasis vesical mediante una talla perineal por su gran volumen. Sin embargo esta técnica tuvo muchos detractores ${ }^{8,11}$.

En las misma época Francisco Díaz escribe su "Tratado nuevamente impreso de las enfermedades de riñones, vejiga y carnosidades de la verga" en 1588. Este texto consta de tres tomos. El primero y el segundo tratan de la litiasis renal y vesical y el tercero hace referencia a las enfermedades de la uretra. Merece mención especial este último porque Francisco Díaz fue el primer autor que describe la técnica de uretrotomía interna y cervicotomía, con una sonda con un borde afilado. En el tomo que hace referencia a la litiasis vesical describe la técnica de Celso y Pierre Franco para la litotomía ${ }^{3}$.

Mariano Santos, discípulo de Giovanni da Romana se encarga en el S. XVI de hacer en sus textos una compilación de las diferentes técnicas quirúrgicas descritas hasta el momento ${ }^{9}$.

El S. XVII viene marcado por la presencia de un personaje llamado Freire Jacques, un fraile que realizó de manera ambulante hasta 5.000 litotomías.

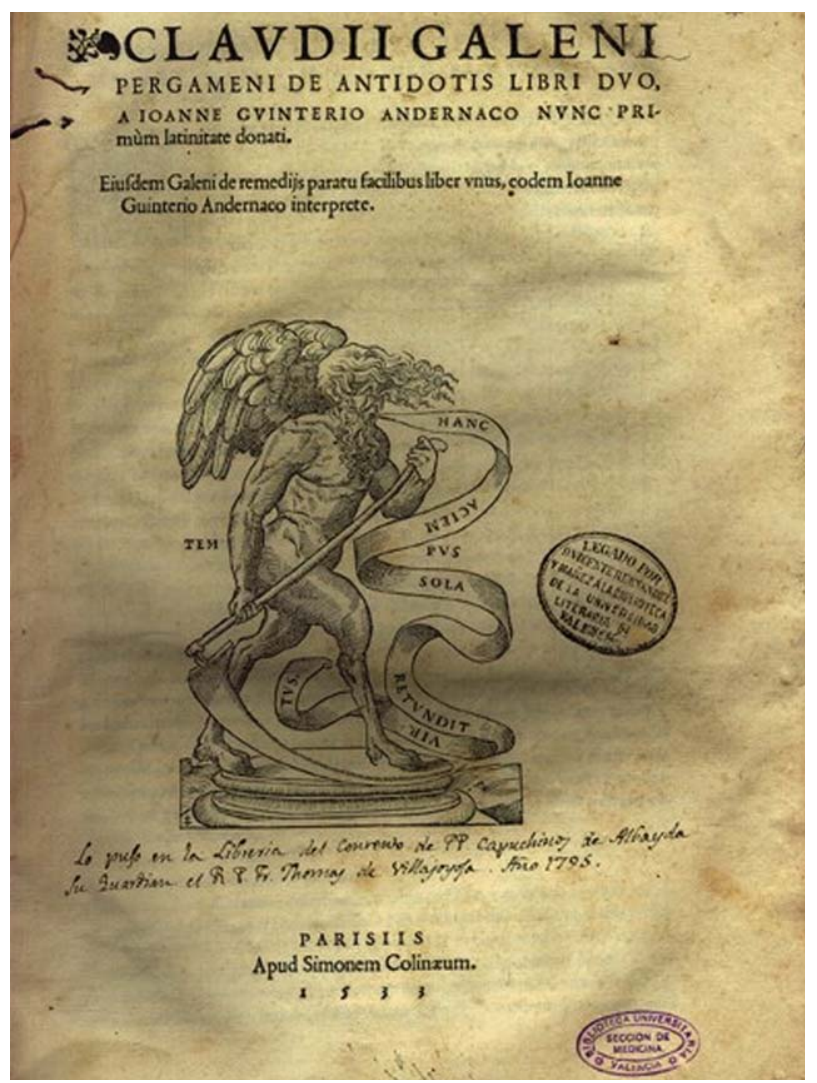

FIGURA 2. Galeno en base a los estudios previos de Celso e Hipócrates describe la técnica de litotricia. 


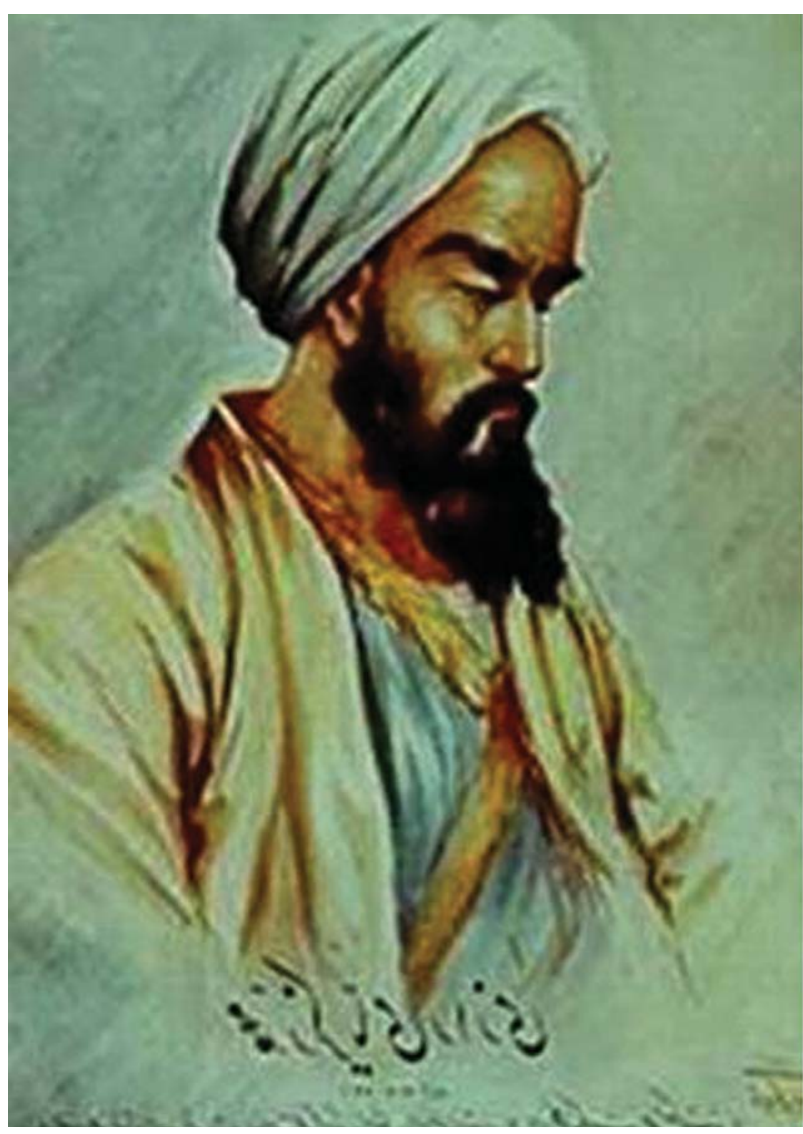

FIGURA 3. Abulcasis fue un gran litotomista del siglo S.X. Fue uno de los primeros en realizar la talla vesical en mujeres.

La litotomía suprapúbica vuelve a tener su auge en el S. XVIII de la mano de Cheselden y Douglas que la pusieron de moda ayudados por el desarrollo de la anestesia de Andrew Morton ${ }^{1,4-6}$.

En el S. XIX destaca la figura de Civiale (Figs. 4a, 4 b y 4c) y Bigelow padres de la técnica de litopalaxia, la primera litotricia endoscópica moderna. En 1827 Civiale publica su memorando de 47 casos realizados con un instrumento llamado "trilabe" que fue modificado en $1833^{1,4,5}$.

En esta época (1813) también describieron experimentos en los que se intentaba disolver la litiasis en el interior de la vejiga con diferentes sustancias (jugos gástricos, ácido clorhídrico, bicarbonato, etc...) aunque no llegaron a probarse en humanos ${ }^{4,5,11}$.

En España Enrique Suender publicó su serie de 220 casos.

Sólo en desarrollo de la endoscópica de Nitze (Fig. 5) mejorará los resultados de un procedimiento que logró desbancar la talla perineal ${ }^{10,12}$.

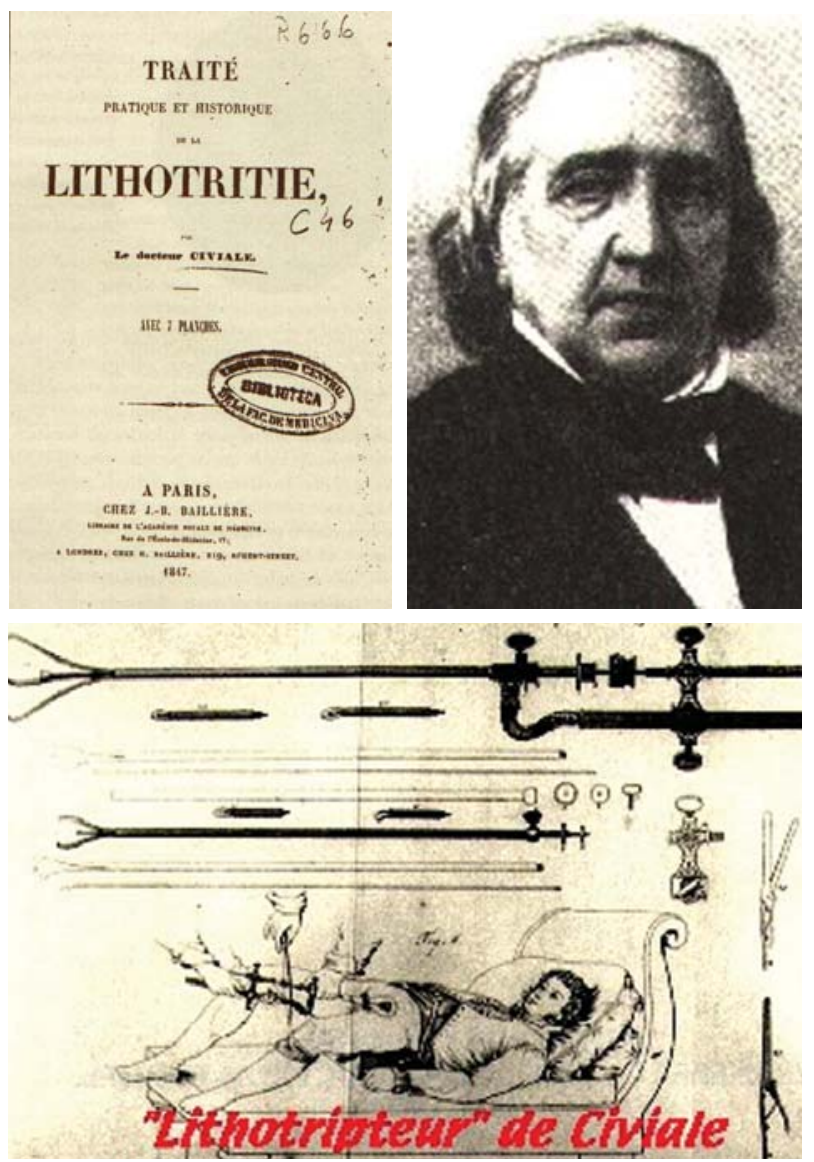

FIGURAS 4 a, b y c. Civiale y Bigelow en el S. XIX describieron la técnica de litopalaxia publicando sus primeras series.

Pero sin duda el gran avance en el tratamiento de la litiasis vesical apareció después de la $2^{\text {a }}$ guerra Mundial. En las batallas se observaba el fenómeno de estallido pulmonar en soldados cerca de la zona de explosión de cargas de profundidad. Este fenómeno fue explicado en 1950 por Jutkin (Fig. 6), de manera que platos de porcelana estallaban bajo el efecto de una onda sónica. En 1953 este autor construye URAT capaz de romper cálculos en vejiga por ondas de ultrasonidos ${ }^{7,8}$.

En 1963 Dornier mejora el aparato y crea ondas de choque que atraviesan los tejidos sin lesionarlos y fue probado inicialmente en perros y posteriormente en humano. Este año llegó a la clínica Dexeus el primer equipo de litotricia extracorpórea de España. La primera litotricia sin anestesia, sin embargo, fue realizada en la Fundació Puigvert (Fig. 7) en los años 70.

La utilización en las litiasis vesicales de la litotricia no ha tardado en imponerse salvo en aquellos 
casos en los que por tamaño o dureza no es posible. En este caso la cistolitotomía vía hipogástrica continua vigente en nuestros días (aunque cada vez menos).

El futuro sin embargo se intuye en las nuevas tecnologías y la terapia génica en aquellos casos en los que sea posible demostrar alteraciones.
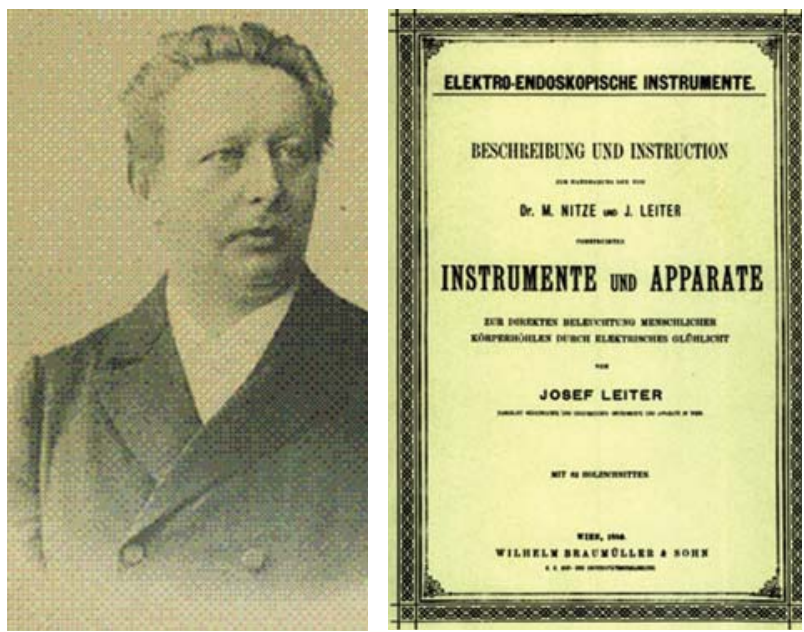

FIGURA 5. Nitze desarrolla en el S.XIX un aparato para explorar cavidades "oscuras" que pronto se aplicó a la Urologia.

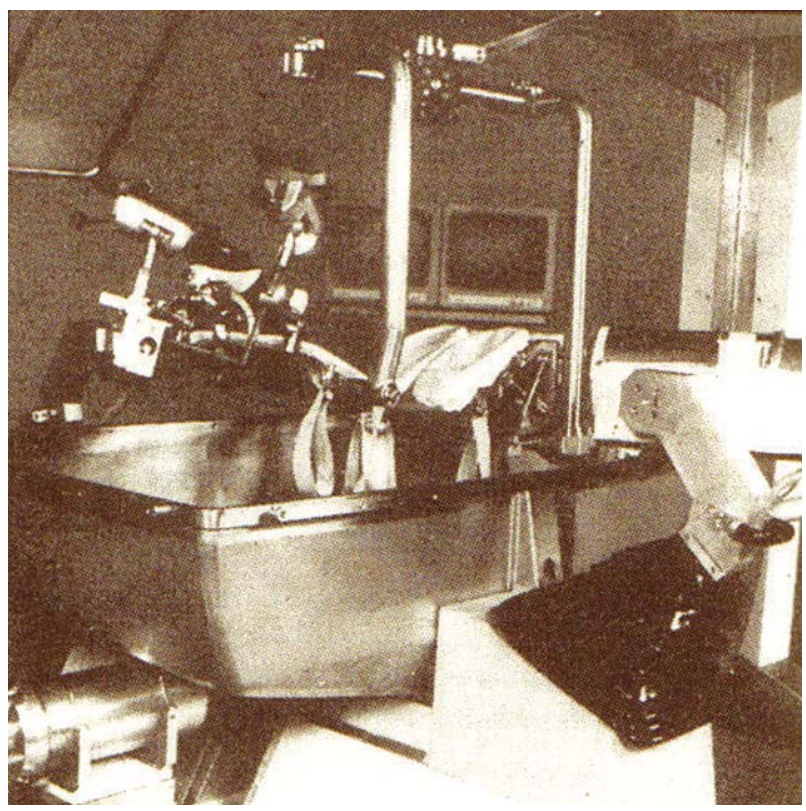

FIGURA 6. El desarrollo de la primera máquina de litotricia fue el punto de partida para los grandes avances del S. XX.

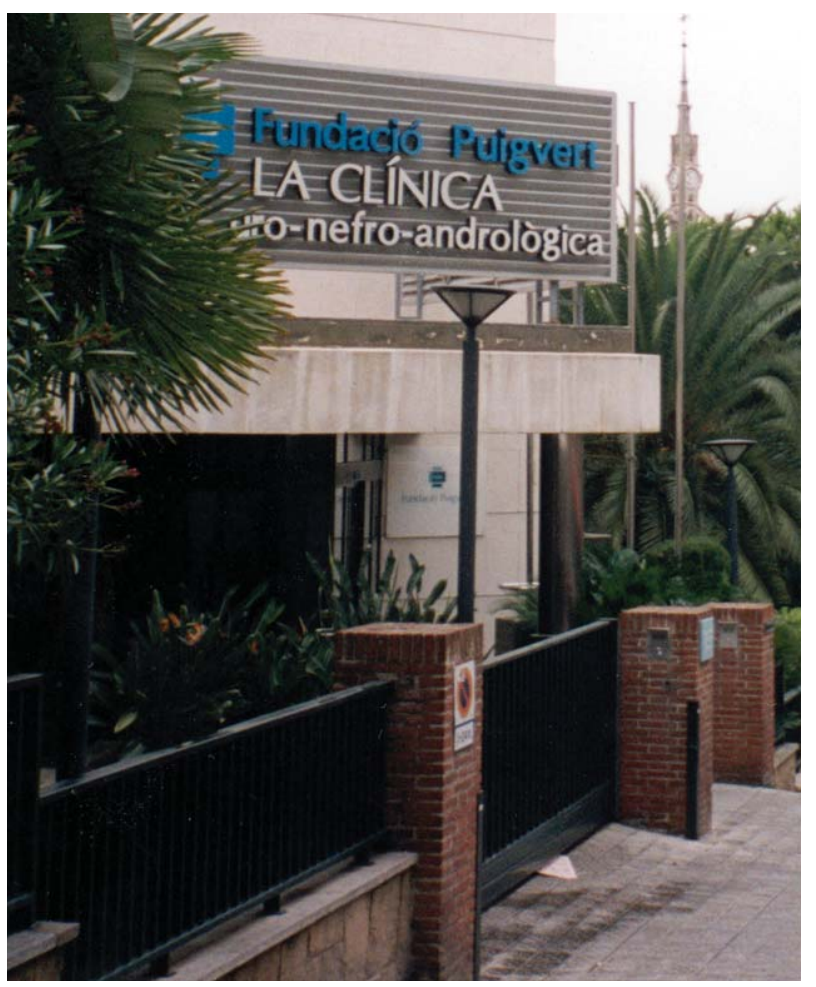

FIGURA 7. La primera litotricia sin anestesia se realizó en Barcelona.

\section{REFERENCIAS}

1. Keyets EL. Enfermedades de los órganos genitourinarios. 1893;323-337.

2. Julián Gutiérrez de Toledo y su Libro "Cura de la piedra y dolor de la yjada o/y cólica renal en 1488". Actas Urol Esp 1994; 18:165-177.

3. Puigvert A. Enfermedades de los riñones, la verga y carnosidades de la vejiga by Francisco Díaz. European Urology 1978;4:232-235

4. Tillaux P. Tratado de cirugía clínica. 1917;3:228-234.

5. Fort JA.. Tratado de patología y clínica quirúrgica. 1875;3: 3567.

6. Lefort L. Manual de medicina operatoria. 1889;627-641.

7. Pérez Castro E. Litolisis en Urología. Arch Esp Urol 1967; Número extraordinario.

8. Actas del $2^{\circ}$ Congreso Español de Historia de la Medicina. 1965; septiembre.

9. Martín Santos L. Barberos y cirujanos de los siglos XVI y XVII. 2000.

10. Marion. Tratado de Urología. 1:591-623.

11. L'Énciclopedie Française. Ed. Dr. E. Desnos. 1914; 3:255-280.

12. Guyon F. Leçons cliniques sur les maladies des voies urinaires. 1897;3:90-103.

Dra. C. Sevilla Cecilia

C/ Agustina de Aragón no 2; 7oA - 06004 Badajoz e-mail: cscuro3@mixmail.com

(Trabajo recibido el 2 de febrero de 2005) 